verter at the coude spectrograph of the 120 -inch reflector. It would be of great value if this or similar equipment could be applied to central regions of barred spirals. I am not aware that Walker has made any measurements of barred spirals.

Ambartsumian: How large are the velocities of streaming you have observed in barred spirals? Have you observed the discontinuity of radial velocity near the central part of the bar?

de Vaucouleurs: The actual values $(\sim 50 \mathrm{~km} / \mathrm{sec})$ are difficult to determine because of scale problems - the scale at the prime focus of the 82 -inch is very small for this work. However, the lines are definitely inclined along the bar. This evidence will be presented tomorrow.

Thackeray: Did I understand you to say that you have cases of a bar pointing directly towards us and, if so, how do you know this?

de Vaucouleurs: Yes, there are definite cases such as NGC 55 and 4631, which are indicated by studies of luminosity distribution and spectrographic observations (see Ap.J. 133 : 405-12 (1961)).

Blaauw: Stating that the observed velocities along the bar are outward, not inward, implies that you know the direction of tilt of the system. Is that well established?

de Vaucouleurs: In some, the direction of tilt is clearly indicated; in others, one can argue about the sign. In edge-on systems, the question does not arise.

\title{
47. DISTRIBUTION DES VITESSES RADIALES DES REGIONS HII DANS LA DIRECTION DU CENTRE GALACTIQUE
}

\author{
G. Courtes
}

Observatoire de Marseille

\section{Introduction}

La Station Française de Zeekoegat (Province du Cap-Afrique du Sud), construite dans le cadre de l'Observatoire Européen Austral est consacrée à l'étude des vitesses radiales par le prisme objectif de Fehrenbach. Parallèlement aux observations du prisme objectif, nous avons, avec P. Cruvellier, conduit un programme d'observations des régions HII de l'Hémisphère Sud.

(a) L'intérêt essentiel des recherches de régions HII est, du point de vue des méthodes d'observation:

$1^{\circ}$-leur caractère monochromatique (émission $\mathrm{H} \alpha$ ),

$2^{\circ}$-leur diamètre apparent considérable (surfaces étendues).

Le premier de ces caractères autorise une sélection aussi grande que l'on veut avec une élimination quasi parfaite du continu.

Le deuxième offre la possibilité d'utiliser les plus grands rapports d'ouverture et d'augmenter ainsi dans un facteur considérable l'éclairement de la plaque.

L'exploitation de ces deux caractères 1 et 2 donne la possibilité de découvrir des régions HII extrêmement faibles ou très absorbées. Le caractère monochromatique donne la possibilité de connaître avec une grande précision la vitesse radiale et les mouvements internes des régions HII; grâce à l'utilisation de l'étalon interférentiel de Perot et Fabry.

(b) Du point de vue purement astrophysique, l'intérêt essentiel des régions HII est leur remarquable répartition régulière le long des bras spiraux (M33, M31, M51, etc. . . .) qui en font l'un des meilleurs guides dans les recherches de structure de la Galaxie, comme l'avaient si bien remarqué Baade et Morgan (Courtès 1962b, 1963b). 
La détermination interférentielle des vitesses radiales des régions HII a montré que leur comportement cinématique était semblable à celui des autres composants de la matière interstellaire (raie de $21 \mathrm{~cm}$ des régions $\mathrm{HI}$, raies d'absorption du sodium et du calcium). Le travail qui suit est le résumé, avant sa publication par $P$. Cruvellier, d'une étude analogue étendue aux régions les plus intéressantes du ciel austral.

\section{Observations de Nouvelles Régions HII dans les Nuages de Magellan et dans la Voie Lactée}

Un petit instrument réfracteur de $20 \mathrm{~cm}$ de diamètre équipé d'un réducteur focal ouvert à $F / 0,95$ a été monté sur la monture de l'instrument destiné aux vitesses radiales stellaires. Ce petit appareil, dont la distance focale équivalente n'était que de $20 \mathrm{~cm}$ nous a permis néanmoins d'avancer certaines connaissances sur l'hydrogène en émission dans les Nuages de Magellan et dans la Voie Lactée.
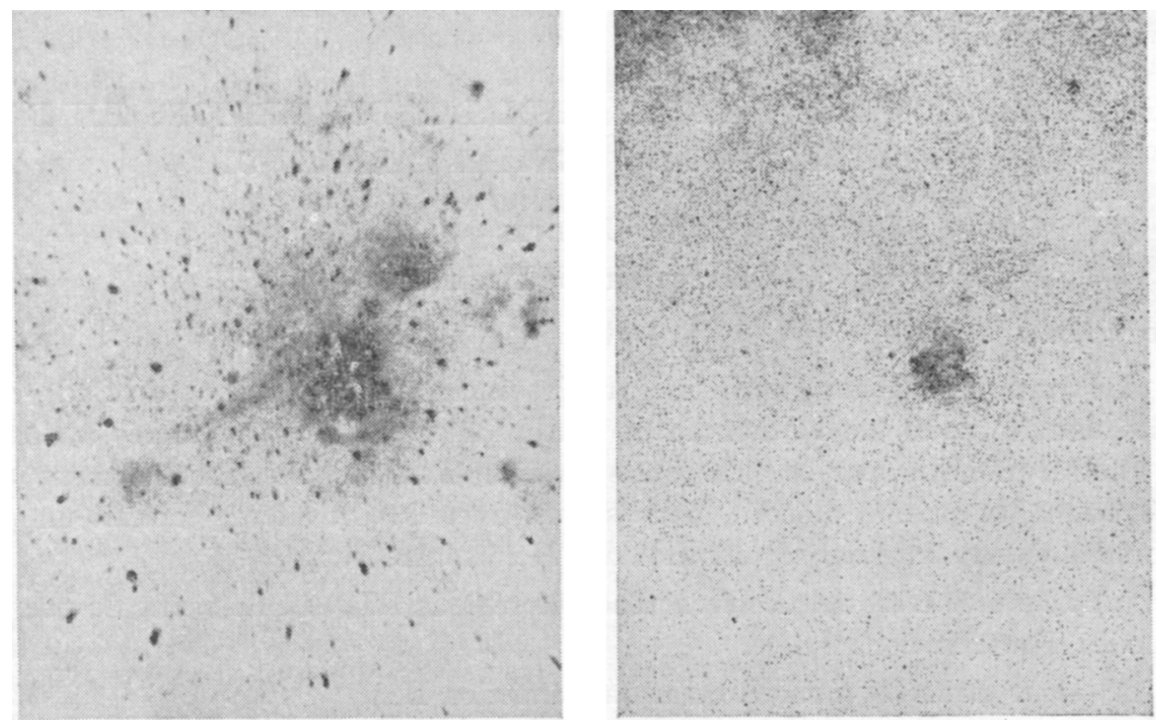

Fig. 1.-Régions centrales de la Galaxie (E12, E13, etc.). A gauche: Réducteur focal $F / 0,95$. Filtre interférentiel $H_{\alpha}(4 \AA)$. Pose h 4. (Photo: P. Cruvellier.) A droite: Reproduction à la même échelle de la même région obtenue avec le Schmidt du Palomar.

La première partie du programme était consacrée à la photographie avec le plus grand contraste possible des régions HII faibles. Le filtre sélectif était un filtre interférentiel de haute qualité de $5 \mathrm{~cm}$ de diamètre et ayant une bande passante de $4 \AA$ centrée sur $\mathrm{H} \alpha$. Ce filtre, dont la transparence était particulièrement bonne $(\mathscr{T}=0,40)$, a été réalisé par les Ets. Grubb Parsons. Le montage particulier de ces filtres à bandes passantes très étroites a été décrit par ailleurs, et je ne donnerai ici que les résultats des observations (Courtès 1960, 1962a, 1963a).

Pour mieux préciser toutefois les qualités d'un tel système, je le comparerai aux filtres colorés qui, jusqu'à présent, ont été les seuls utilisés avec les appareils de distance focale de l'ordre de $20 \mathrm{~cm}$. 
Un filtre de $4 \AA$ a une bande passante 60 fois plus étroite environ que celle des filtres colorés de verre ou de gélatine.

A condition d'atteindre le voile de fond de ciel de l'émulsion photographique, ce qui nécessite 2 à 3 heures de pose, on est certain avec de tels filtres, de détecter des nébuleuses 60 fois moins brillantes que celles qui avaient été détectées jusqu'à présent. Ce facteur de contraste est particulièrement appréciable lorsque le champ est très encombré d'étoiles. C'est là, comme tous les spécialistes de cette question le savent, une très grande difficulté de détection des régions HII faibles dans la Voie Lactée.

Pour donner un exemple bien connu du progrès réalisé par ce genre d'observations, nous donnons reproduite ci-contre une reproduction de la Palomar Sky Survey concernant les régions très voisines du centre galactique (Fig. 1).

On reconnaît facilement sur ce cliché la région HII, E13 et plus difficilement E15, E16, cataloguées par Sharpless, et en regard, le même champ obtenu avec notre appareil à la même échelle.

On voit non seulement apparaître très distinctement les nébuleuses du Catalogue de Sharpless, mais encore de nombreuses régions nouvelles qui donnent à ce champ un aspect tout différent de celui de la Palomar Sky Survey.

Evidemment, la qualité des images des étoiles est bien inférieure sur notre cliché en raison de la très petite distance focale: $20 \mathrm{~cm}$ pour notre appareil, en comparaison de la distance focale de $3 \mathrm{~m} \mathrm{du}$ Schmidt du Mont Palomar. La discussion du meilleur rapport d'ouverture et de la meilleure distance focale à utiliser pour détecter les régions HII est donc à réviser.

On se rappelle qu'au Congrès de Moscou, cette discussion sous la présidence de B. Bok avait conduit $H$. Johnson, van de Hulst, Sharpless, et moi-même à la conclusion que le 48-inch, grâce à sa distance focale, constituait le meilleur détecteur, en dépit de son faible rapport d'ouverture $(2,5)$ et de la sélectivité insuffisante des filtres colorés. On voit, qu'en fait, c'est la sélectivité du filtre qui l'emporte de beaucoup dans la sensibilité de détection des régions HII.

Un travail analogue était nécessaire dans l'ensemble de la Voie Lactée et principalement, dans les régions situées de part et d'autre de la direction du centre galactique.

On a déjà pu comparer par l'exemple de la Palomar Sky Survey l'enrichissement d'information que l'on a pu obtenir dans la région du centre galactique.

Nous avons voulu surtout dégager la nébuleuse Stromlo $134 \mathrm{du}$ riche nuage d'étoiles du Sagittaire. Cette nébuleuse avait été mise en évidence au cours des premières observations avec filtres interférentiels faites en 1950 a l'Observatoire de Haute-Provence (Courtès 1962c) d'après les conseils de Strömgren, puis par Strömgren et Johnson, et enfin, définitivement catalogués dans l'atlas publié par A. W. Rodgers et al. (1960), à la suite des observations de la caméra à champ plan de Meinel.

La photographie jointe ici montre Stromlo 134 complètement dépouillée $\mathrm{du}$ fond stellaire et l'on remarque en particulier, la décroissance générale de sa brillance en $\mathrm{H}_{\alpha}$ au fur et à mesure de la pénétration dans le Grand Nuage du Sagittaire (Fig. 2). 
On pourrait donner d'autres exemples encore de ce genre de photographie, mais nous préférons attendre d'avoir une étude complète de la Voie Lactée dans l'Hémisphère Nord et dans l'Hémisphère Sud pour publier un Atlas aussi détaillé que possible des régions HII faibles qui n'ont pas encore été découvertes, ou qui sont mal dégagées des nuages d'étoiles.

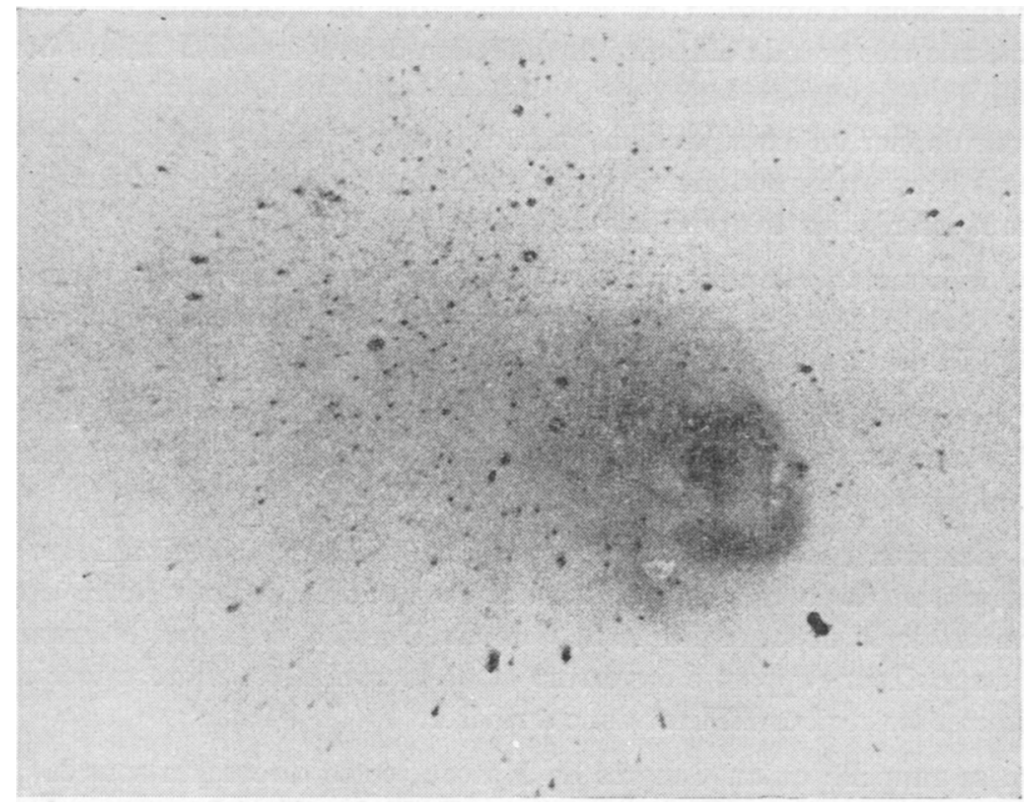

Fig. 2.-Stromlo 134-Pose 3 h. Plaque Kodak 103 aE. Filtre interférentiel H $\alpha$ (4 $\AA$ ). (Photo: P. Cruvellier.)

\section{Analyse des Vitesses Radiales des Régions HII entre 270 et $30^{\circ}$ de Longitudes Galactiques}

$\mathrm{Au}$ cours d'une première série d'observations faites en juillet et août 1959 au Royal Observatory du Cap de Bonne Espérance, j'avais pu obtenir la vitesse radiale d'une série de régions HII situées le long du plan galactique et assez proches de la direction du centre galactique pour mettre en évidence la variation et le changement de signe de la vitesse radiale de l'hydrogène interstellaire au moment du passage de part et d'autre de la direction du centre galactique.

Ce travail avait surtout pour but de voir si les vitesses s'annulaient effectivement dans la direction du centre comme le voudrait la théorie de la rotation différentielle de la Galaxie.

La première série de mesures faites à Cape Town a donné une répartition de l'ensemble des régions HII telle que le point où la vitesse s'annule se trouve à quelques degrés Sud du centre galactique et non pas dans la direction même du centre.

Le diagramme ci-contre où l'on a porté la vitesse radiale en ordonnée et la longitude galactique en abscisse montre la répartition des points observés. 
Dans cette étude, si l'on fait abstraction des connaissances assez incertaines d'ailleurs, de la distance des nébuleuses, on voit que la répartition des points se rapproche d'une droite parallèle à la courbe de la rotation différentielle correspondant à une distance de l'ordre de 3 kpc (Fig. 3).

Il est évident que cette distance n'est certainement pas celle de toutes les nébuleuses portées sur le graphique.

Depuis cette étude et la fondation de la Station de Zeekoegat, P. Cruvellier a observé systématiquement la Voie Lactée du Sud avec le même appareil que celui que j'ai décrit précédemment.

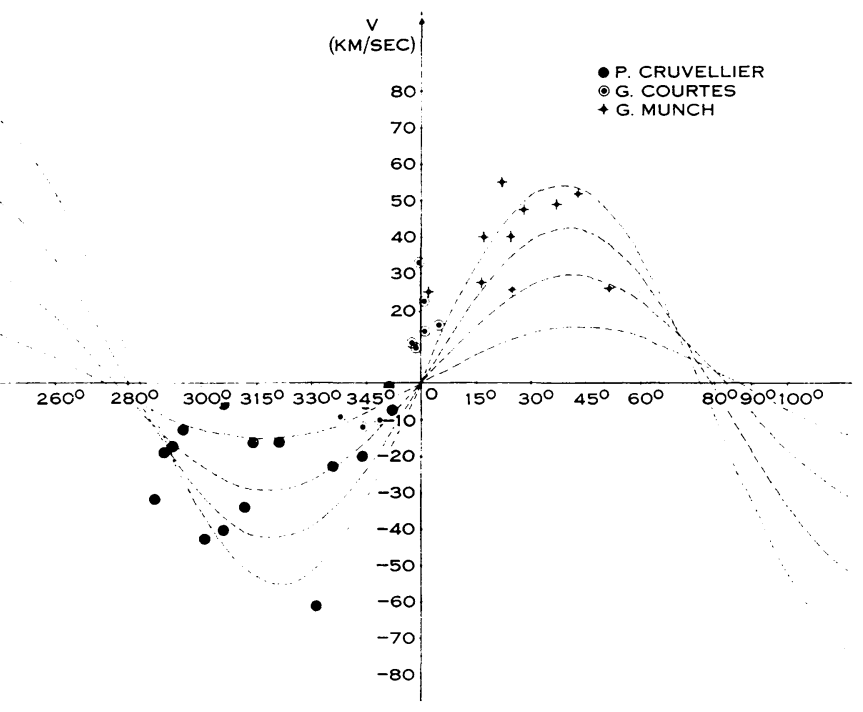

Fig. 3.-Repartition des vitesses radiales des régions HII dans les directions environnant le centre galactique. (Mesure interférentielle de la radiation $\mathbf{H} \alpha$.)

La Figure 3 résume les anciens et les nouveaux résultats obtenus. Il semble se dégager une répartition des vitesses en deux bras spiraux dans la région comprise entre 300 et $340^{\circ}$ de longitude galactique, avec des vitesses radiales de $-15 \mathrm{~km} / \mathrm{sec}$ pour le bras le plus proche et de $-40 \mathrm{~km} / \mathrm{sec}$ pour le bras le plus éloigné.

$\mathrm{Au}$ cours de la mission du Royal Observatory en 1959, j'ai accordé une attention toute particulière à la région centrale de la Galaxie (Fig. 1). II n'est pas déraisonnable en effet, de rechercher des régions HII près du noyau d'une nébuleuse spirale comme la Galaxie, de nombreux exemples nous montrent d'intenses régions HII dans les régions centrales des galaxies. Je ne citerai que M33 comme exemple, la photographie publiée au cours du même Symposium (Régions HII dans les Nuages de Magellan et les galaxies proches), ayant été prise avec le réducteur focal et un filtre de $4 \AA$ à bandes passantes, à l'aide du télescope de $193 \mathrm{~cm}$ de l'Observatoire de Haute-Provence. On y voit, près du centre, dans un rayon de $1 \mathrm{kpc}$ d'intenses régions HII dégagées ici pour la première fois des intenses nuages d'étoiles qui empêchent de les détecter sur des photographies ordinaires (Courtès 1962b). 
La méthode interférentielle est de plus, un moyen extrêmement efficace pour détecter le faible taux de lumière monochromatique transmis par un nuage absorbant très dense. Si l'on a pu penser que le 48-inch muni de filtres colorés de $250 \AA$ de bande passante pouvait éventuellement montrer de faibles plages d'émission, cet espoir est encore plus justifié avec le couplage filtre interférentiel-étalon.

Le Tableau 1 résume les performances que l'on peut atteindre.

Tablead I

LIMITE DE DETECTION D'UNE SOURCE ÉTENDUE ET MONOCHROMATIQUE

\begin{tabular}{|c|c|c|c|c|c|}
\hline & Filtre $250 \AA$ & Filtre $50 \AA$ & Filtre $4 \AA$ & $\begin{array}{l}\text { Filtre } 50 \AA \\
+ \text { Etalon } \\
5 \text { couches }\end{array}$ & $\begin{array}{l}\text { Filtre } 4 \AA \\
+ \text { Etalon } \\
5 \text { couches }\end{array}$ \\
\hline $\begin{array}{l}\text { Intensite d'une } \\
\text { région HII juste } \\
\text { détectable }\end{array}$ & $I$ & $0,20 I$ & $0,016 I$ & $0,013 I$ & $0,001 I$ \\
\hline$F / 2,5$ & $1 \mathrm{~h}$ & $5 \mathrm{~h}$ & $62 \mathrm{~h}$ & $75 \mathrm{~h}$ & $1000 \mathrm{~h}$ \\
\hline$F / 0,8$ & $6 \mathrm{~m}$ & $30 \mathrm{~m}$ & $6 \mathrm{~h}$ & $7 \mathrm{~h}$ & $100 \mathrm{~h}$ \\
\hline
\end{tabular}

Note: Les durées de pose évaluées n'ont qu'un caractère grossièrement indicatif. La dernière colonne justifie l'emploi de la caméra electronique.

Une région HII 75 fois plus faible que celles à la limite de détection de la Palomar Sky Survey serait mise en évidence (Col. 4) ou si l'on préfère, une région HII à la limite du 48 inch se signalerait de la même façon à travers une absorption de 4 magnitudes.

L'étude en lumière infra-rouge de Hugh Johnson faite au Mount Stromlo il y a quelques années, avait incité son auteur à me demander d'essayer de déterminer la vitesse radiale des nébuleuses $\mathrm{E} 12, \mathrm{E} 13$, etc. . . . Ces nébuleuses ont une remarquable disposition autour du centre galactique, radio source, Sagittaire A; une telle coincidence est si improbable que l'on est en droit de se demander si E12, E13, etc. ne sont pas en relation avec les régions $H I$ réellement situées au centre de la Galaxie, et animées d'un fort mouvement de rotation et d'expansion comme l'ont découvert Oort et Rougoor. En dépit de cette coïncidence assez exceptionnelle, la morphologie de E13 telle qu'elle est donnée par la photographie de la Palomar Sky Survey laissait peu de chances à cette interprétation comme Johnson le reconnaissait lui-même, mais les autres nébuleuses plus faibles auraient pu éventuellement être rattachées aux régions profondes du centre et une observation spécifique n'était pas inutile.

Dans l'hypothèse où ces régions auraient fait partie du disque de région $\mathrm{HI}$ découvert avec le télescope de Dwingeloo, on devait s'attendre à ce que leurs vitesses radiales soient de l'ordre de grandeur de celles trouvées par Oort et Rougoor, c'est-àdire -100 à $-200 \mathrm{~km}$ à la seconde.

Dans les autres régions de la Voie Lactée, tout particulièrement le long des bras spiraux d'Orion et de Persée, la corrélation: Vitesse radiale $\mathbf{H} \alpha$-vitesse radiale $21 \mathrm{~cm}$, est particulièrement bonne et par conséquent, rend tout à fait raisonnable 
cette hypothèse. Les belles observations de la vitesse d'expansion des raies 37263729 de [OII] par G. Münch autour du noyau de M31, confirmaient encore l'intérêt de ces recherches.

Nous nous sommes donc efforcés de mesurer la vitesse radiale des nébuleuses E12, E13 etc. avec la méthode interférentielle et nous avons obtenu les résultats suivants qui ont d'ailleurs déjà été publiés lors du Symposium International de Paris (Courtès et Cruvellier 1960a).

On voit que (Tableau II) les vitesses radiales se rangent entre +13 et $+31 \mathrm{~km} /$ sec et semblent n'avoir, par conséquent, aucun rapport avec la vitesse radiale des régions centrales.

\section{Tableau II}

VITESSES RADIALES DES NEBULEUSES

\begin{tabular}{c|c}
$\begin{array}{c}\text { Nébuleuse } \\
\text { Catalogue Sharpless }\end{array}$ & $\begin{array}{c}\text { Vitesse Radiale } \\
(\mathrm{km} / \mathrm{sec})\end{array}$ \\
\hline $\mathrm{E} 13$ & +13 \\
$\mathrm{E} 15$ & +24 \\
$\mathrm{E} 16$ & +31 \\
\hline
\end{tabular}

Par contre, ces vitesses de 13 à $31 \mathrm{~km} / \mathrm{sec}$ s'interpolent très bien dans l'ensemble des mesures obtenues pour les autres régions voisines le long de la Voie Lactée.

On peut donc raisonnablement considérer qu'elles font partie du bras spiral interne et non des régions centrales.

Cependant, au cours de ces recherches, l'appareil que nous utilisions ayant un champ assez grand de $2^{\circ} 30^{\prime}$, une faible émission se remarquait sur le cliché par un fragment d'anneau d'interférence dans les régions suivantes:

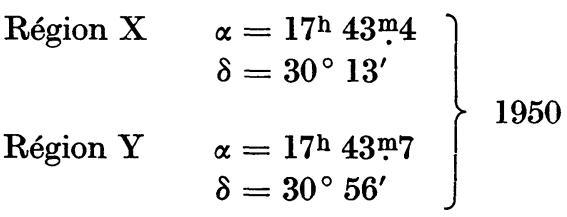

situées à $1^{\circ}$ environ de Sagittaire A.

La mesure de la vitesse radiale de ce faible fragment d'anneau montre une vitesse radiale de l'ordre de $-150 \mathrm{~km} / \mathrm{sec}$, qui pouvait être attribuée aux régions centrales. La région en question se trouve dans la zone de transparence relative, signalée par Hugh Johnson à la suite de ses recherches en lumière infra-rouge, et, par conséquent, il n'y a pas d'incompatibilité absolue à interpréter ces résultats comme provenant d'une région lointaine. Ce n'est que l'année suivante que nous avons pu observer à nouveau avec un grand pouvoir séparateur (10 fois plus grand) grâce au télescope de 1,93 m de l'Observatoire de Haute-Provence.

$\mathrm{Au}$ cours de deux nuits particulièrement transparentes, malgré la hauteur faible du centre galactique à la latitude de l'Observatoire de Haute-Provence nous 
avons pu obtenir, M. Cruvellier et moi-même, deux clichés convenablement exposés de deux régions choisies aux endroits les plus intenses du faible anneau trouvé au Cap (Courtès et Cruvellier 1960b).

La reproduction ci-contre montre l'un de ces clichés comparé aux anneaux d'étalonnage d'un tube à hydrogène et aux anneaux d'étalonnage de la Nébuleuse M8, dont la vitesse radiale est três faible (moins $7 \mathrm{~km} / \mathrm{sec}$ ) (Fig. 4).

Sur ce cliché, une inter-frange correspond à $395 \mathrm{~km} / \mathrm{sec}$.

Le décalage entre les anneaux de M8 et de $\mathrm{H}_{\alpha}$ est, de ce fait, inappréciable sans comparateur. On remarque entre les anneaux de $\mathrm{H} \alpha$ ceux de la raie 6584 de

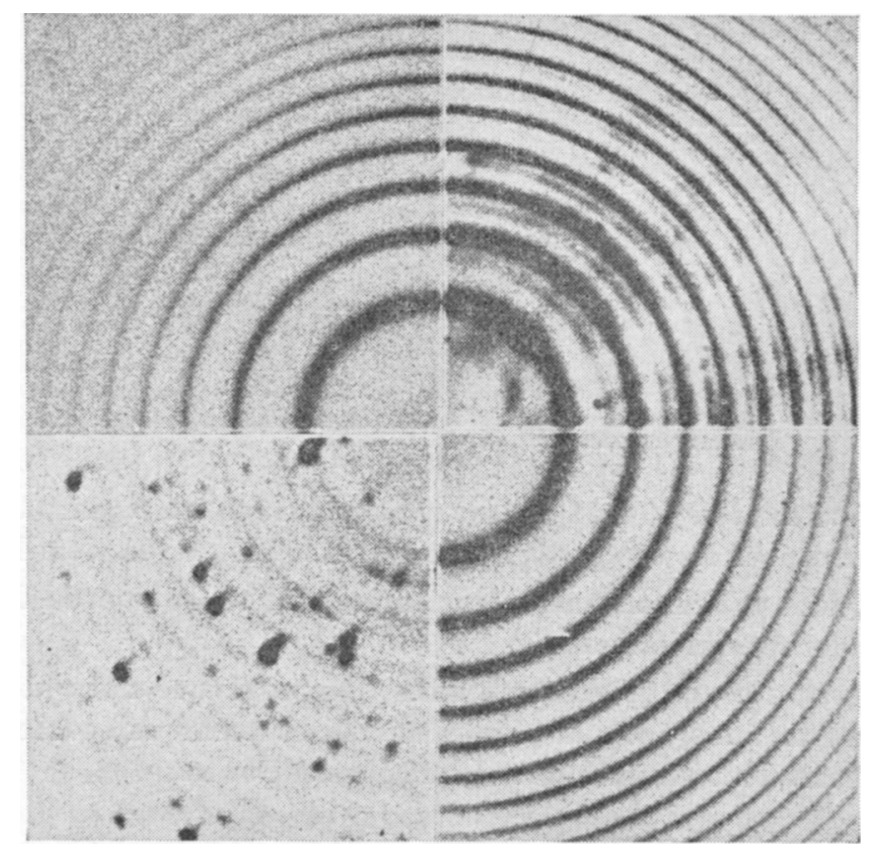

Fig. 4.-Détection optique de l'hydrogène ionisé dans la direction du bulbe central de la Galaxie. Le montage photographique montre la comparaison d'anneaux de Perot et Fabry donnés par la radiation $\mathrm{H} \alpha .1^{\circ}$-Tube à hydrogène (en haut et à gauche et à droite et en bas). $2^{\circ}$-Nébuleuse M8 (en bas et à gauche). $3^{\circ}$-Région située dans la direction du bulbe central de la Galaxie. $\alpha=17^{\mathrm{h}} 43^{\mathrm{m}} 4, \delta=-30^{\circ} 13^{\prime}$ (en haut et à droite). Télescope $193 \mathrm{~cm}$. Réducteur focal $F / 0,95$. Filtre interférentiel $60 \AA$. Centre galactique. Pose 3 h. Plaque 103aE Kodak. M8. Pose $10 \mathrm{~m}$. (G. Courtès-P. Cruvellier.)

[NII], l'ordre d'interférence de l'étalon interférentiel ayant été choisi de telle façon que le décalage entre $\mathrm{H} \alpha$ et [NII] soit suffisant.

La partie du cliché correspondant à la région $\mathrm{X}$ montre un décalage systématique d'anneaux plus faibles appartenant à $\mathrm{H} \alpha$ et à [NII]. On constate que le déplacement est de l'ordre de grandeur d'une demi inter-frange, c'est-à-dire, après que les mesures aient été effectuées $-188 \mathrm{~km} / \mathrm{sec}$. 
Il nous a semblé que ces deux résultats obtenus à St Michel pouvaient représenter effectivement le très faible signal d'une région HII du disque central des régions $\mathrm{HI}$ découvertes à Dwingeloo.

Une vérification supplémentaire fut faite en mesurant très soigneusement le déphasage entre les raies de $\mathrm{H} \alpha$ et $6584 \AA$ sur le cliché correspondant à la région $\mathrm{X}$, ce déphasage s'est montré identique à celui que l'on pouvait attendre par le calcul et par l'observation sur d'autres régions présentant simultanément ces deux émissions et cela, avec une précision de $1 / 1000 \mathrm{e}$ d'inter-frange près. Ces vérifications pourraient paraître suffisantes mais la complexité du rayonnement du ciel nocturne laisse un certain doute sur cette interprétation; le filtre interférentiel qui sélectionnait le domaine spectral utilisé avait une bande passante de $50 \AA$ et une partie des bandes de $\mathrm{OH}$ était transmise par ce filtre.

Bien que nous ayions fait une étude préliminaire très soigneuse de l'influence des bandes de $\mathrm{OH}$ dans la détection interférentielle des régions $\mathrm{HII}$, et bien que l'intensité de ces bandes soit très faible en été (mois d'août), il restait encore cette indétermination dans l'interprétation des résultats. Nous avons alors fait au cours de la même période d'observation un interférogramme de la nébuleuse Stromlo 134 située à une distance zénithale plus forte encore que celle de la région $\mathrm{X}$. Aucune émission de $\mathrm{OH}$ n'a été détectée sur ce cliché.

Nous comptions, au cours de notre mission de 1962 en Afrique du Sud, reprendre de nouveaux clichés, mais les conditions atmosphériques ont été totalement défavorables à Cape Town où se trouvait le seul télescope suffisamment puissant pour aborder ce problème.

En conclusion, une étude soignée dans une latitude convenable avec un instrument analogue à celui de l'Observatoire de Haute-Provence est absolument nécessaire pour confirmer ces résultats. S'il en est ainsi, on aura alors atteint optiquement les régions très voisines $\mathrm{du}$ centre galactique.

Pour l'ensemble de ces recherches, nous sommes heureux d'exprimer notre gratitude à Her Majesty's Astronomer Dr. Stoy et Dr. Evans, Director and Chief Assistant at the Royal Observatory, ainsi qu'au Dr. Thackeray, Director of the Radcliffe Observatory, pour l'aide constante qu'ils nous ont apportée.

\section{Discussion}

de Vaucouleurs: What was the size of the area covered by these large negative velocities?

Courtès: It is difficult to say, but at Cape Town the field was approximately $2^{\circ}$ by $30^{\prime}$. The emission area had an area of approximately $1^{\circ}$ by $30^{\prime}$. With the 77 -inch at Haute-Provence we have observed the two brightest parts of this area.

Aller: I would like to express my admiration for Courtès' work on emission nebulae, especially on those in M33. Some years ago, G. Haro used an objective prism and a narrow band-pass filter on the Tonantzintla Schmidt camera to add many emission nebulosities to those already found in the Triangulum spiral. At Michigan, with the Curtis Schmidt, we measured the integrated brightnesses of these nebulosities, but details were not published as $I$ wished to obtain their angular sizes, surface brightnesses, and magnitudes of their illuminating stars. This latter program was quite outside the capabilities of equipment available at Michigan, but I am now hoping to complete this work. Overlap of stellar images near the nucleus of M33 concealed the emission nebulosities which are so neatly revealed by Courtès' filter technique. 


\title{
Bibliographie
}

Courtes, G. (1960).-Ann. d'Astr. 23: 115-217.

Courtes, G. (1962a).-C.R. Acad. Sci. (Paris) 254: 1738-40.

Countes, G. (1962b).-Astronomie 76: 224-31.

Courtes, G. (1962c).-Astronomie 76: 250-8.

Courtes, G. $(1963 a)$.- - Paper presented to Symposium on Instrumental Astronomy at the 113th Meeting of the American Astronomical Society, Tucson, Arizona.

Courtes, G. (1963b).-Astronomie 77 : (in press).

Courtes, G., et Cruverlier, P. (1960a)._- "Les recherches galactiques et extragalactiques et la photographie electronique." = Colloque Int. CNRS no. 95. [Paris 1959.] Ann. d'Astr. 23: 419-21.

Courtss, G., et Cruvellier, P. (1960b).-C.R. Acad. Sci. (Paris) 251 : 2470-2.

Rodgers, A. W., Campbelu, C. T., Whiteoak, J. B., Bailey, H. H., and Hunt, V. O. (1960)."An Atlas of H-alpha Emission in the Southern Milky Way." (Mount Stromlo Observatory: Canberra.)

\section{ON THE HII REGIONS IN GALAXIES}

\author{
J. L. SERSIC* \\ Observatorio Astronomico, Córdoba
}

In the present paper we shall consider that the HII complexes observed in galaxies may be interpreted as the resulting Strömgren volume produced by a cluster or association of exciting stars. If $\rho_{\mathbf{H}}$ is the density of the hydrogen surrounding the cluster or association and $I$ the number of ionizing quanta $(\lambda<912 \AA)$ emitted by the stars, the ionized volume becomes

$$
V_{i} \propto I / \rho_{\mathrm{H}}^{2} \text {. }
$$

It is possible to prove that the number of ionizing quanta $I(L)$ emitted by a star is proportional to the bolometric luminosity $L$ for spectral types earlier than B3, so that, if $\phi(L)$ is the actual luminosity function of the cluster (in stars per cubic parsec) and $V$ a representative volume, then

$$
I \propto V \int_{L_{1}}^{L} \phi(L) L \mathrm{~d} L .
$$

Let us now assume that the present luminosity function results from a universal luminosity function $\psi(L)$ valid for all the galaxies, then the preceding expression becomes

$$
I \propto V \int_{L_{1}}^{L} \mathrm{~d} L \int_{t-\tau(L)}^{t}(\mathrm{~d} N / \mathrm{d} T) \psi(L) L \mathrm{~d} t,
$$

where $L_{1}$ is the luminosity of the B3 stars. Here $\tau(L)$ is the lifetime of the stars with luminosity $L$ in the main sequence. By assuming now a constant creation rate $(\mathrm{d} N / \mathrm{d} t)$ in a short interval such as $\tau(L) \propto M / L$, we have

$$
I \propto V(\mathrm{~d} N / \mathrm{d} T) \int_{L_{1}}^{L} \psi(L) M \mathrm{~d} L,
$$

* Presented by A. W. Rodgers. 